PROCEEDINGS OF THE

AMERICAN MATHEMATICAL SOCIETY

Volume 129, Number 8 , Pages 2431-2439

S 0002-9939(00)05829-9

Article electronically published on December 28, 2000

\title{
FINITENESS OF POLYHEDRAL DECOMPOSITIONS OF CUSPED HYPERBOLIC MANIFOLDS OBTAINED BY THE EPSTEIN-PENNER'S METHOD
}

\author{
HIROTAKA AKIYOSHI \\ (Communicated by Christopher Croke)
}

\begin{abstract}
Epstein and Penner give a canonical method of decomposing a cusped hyperbolic manifold into ideal polyhedra. The decomposition depends on arbitrarily specified weights for the cusps. From the construction, it is rather obvious that there appear at most a finite number of decompositions if the given weights are slightly changed. However, since the space of weights is not compact, it is not clear whether the total number of such decompositions is finite. In this paper we prove that the number of polyhedral decompositions of a cusped hyperbolic manifold obtained by the Epstein-Penner's method is finite.
\end{abstract}

\section{INTRODUCTION}

Let $M$ be a noncompact complete orientable hyperbolic $n$-manifold of finite volume. We call such a manifold a cusped hyperbolic $n$-manifold. In 11, Epstein and Penner give a canonical method of decomposing $M$ into hyperbolic ideal polyhedra. Starting with positive weights, i.e., positive real numbers, specified for the cusps, they apply a convex hull construction in the Minkowsky space. The resultant polyhedral decomposition is unchanged even if the weights are simultaneously multiplied by a positive number.

Suppose that $M$ has two or more cusps. Then the weights can be deformed so that their ratio changes. From the definition, it is rather obvious that such polyhedral decomposition is "stable" with respect to the weights. Namely, only a finite number of polyhedral decompositions appear when the weights are slightly changed. Thus the set of weights admits a locally finite stratification up to scalar multiple, i.e., the set of weights providing the same polyhedral decomposition defines a stratum of the stratification. Since the space of positive weights up to scalar multiple is not compact, it is not clear whether this stratification contains only finitely many members. In this paper, we find a good compactification for it and prove the following finiteness property.

Theorem 1.1. The number of ideal polyhedral decompositions of a given cusped hyperbolic n-manifold $M$ obtained by the Epstein-Penner's method is finite.

Received by the editors May 5, 1999 and, in revised form, December 21, 1999.

2000 Mathematics Subject Classification. Primary 51M10; Secondary 57M50.

Key words and phrases. Hyperbolic manifold, canonical decomposition, ideal polyhedral decomposition. 
By the way, Epstein and Penner further put a natural auxiliary Euclidean structure on each polyhedron of the decompositions and show that such singular Euclidean structures are then projectively parameterized by the weights. However, Theorem 1.1 does not concern this additional structure. What we study for the rest of this paper are the underlying hyperbolic ideal polyhedral decompositions.

\section{Convex hull Construction}

The Minkowsky $(n+1)$-space $\mathbb{M}^{n+1}$ is an $(n+1)$-dimensional real vector space with the bilinear form $\langle\cdot, \cdot\rangle$ of signature $(n, 1)$, i.e.,

$$
\langle x, y\rangle=-x_{0} y_{0}+x_{1} y_{1}+\cdots+x_{n} y_{n},
$$

for $x, y \in \mathbb{M}^{n+1}$ with $x=\left(x_{0}, x_{1}, \ldots, x_{n}\right), y=\left(y_{0}, y_{1}, \ldots, y_{n}\right)$. The hyperbolic $n$-space is the subset of $\mathbb{M}^{n+1}$ defined by

$$
\mathbb{H}^{n}=\left\{x=\left(x_{0}, x_{1}, \ldots, x_{n}\right) \mid\langle x, x\rangle=-1, x_{0}>0\right\}
$$

equipped with the Riemannian metric which is the restriction of $\langle\cdot, \cdot\rangle$ to the tangent spaces.

We define the positive light cone $L_{+}$and the set $T_{+}$by

$$
\begin{aligned}
& L_{+}=\left\{x=\left(x_{0}, x_{1}, \ldots, x_{n}\right) \mid\langle x, x\rangle=0, x_{0}>0\right\} \subset \mathbb{M}^{n+1}, \\
& T_{+}=\left\{x=\left(x_{0}, x_{1}, \ldots, x_{n}\right) \mid\langle x, x\rangle<0, x_{0}>0\right\} \subset \mathbb{M}^{n+1} .
\end{aligned}
$$

For each $v \in L_{+}$, we define the set $H_{v}$ by

$$
H_{v}=\left\{x \in \mathbb{H}^{n} \mid\langle v, x\rangle \geq-1\right\} .
$$

Such $H_{v}$ is called a horoball in $\mathbb{H}^{n}$. We can see that $H_{v}=H_{v^{\prime}}$ implies $v=v^{\prime}$, and hence can associate a vector $v(H)$ with each horoball $H$ in $\mathbb{H}^{n}$ so that $H=H_{v(H)}$. From the definition, the map $v$ from the set of horoballs in $\mathbb{H}^{n}$ to $L_{+}$is bijective.

Suppose that $M$ has $p$ cusps. Let $\pi: \mathbb{H}^{n} \rightarrow M$ be the universal covering and denote by $\Gamma$ the image of $\pi_{1}(M)$ by the holonomy map. To define a decomposition of $M$, we specify a weight, i.e., a nonnegative real number, for each cusp. Let $w_{j}(j=1, \ldots, p)$ be the weight specified for the $j$-th cusp and $W=\left(w_{1}, \ldots, w_{p}\right)$ the collection of them, which we also call simply a weight. Suppose that $W$ is an element of $\left(\mathbb{R}_{\geq 0}\right)^{p}-\{\mathbf{0}\}$, where $\mathbb{R}_{\geq 0}$ is the set of nonnegative real numbers and $\mathbf{0}$ denotes the origin of $\mathbb{R}^{p}$. Generalizing a convex hull construction of Epstein-Penner [1], we define the decomposition $\mathcal{D}(W)$ of $M$ with weight $W$ as follows. Let $H_{j}$ $(j=1, \ldots, p)$ be a horoball with the following properties: (i) $H_{j}$ is stabilized by a parabolic subgroup of $\Gamma$ which corresponds to the $j$-th cusp. (ii) The volume of $H_{j} / \operatorname{Stab}\left(\Gamma, H_{j}\right)$ is equal to $w_{j}$, where $\operatorname{Stab}\left(\Gamma, H_{j}\right)$ denotes the subgroup of $\Gamma$ which stabilizes $H_{j}$. (When $w_{j}=0$, put $H_{j}=\emptyset$.) Then we define the following sets:

$$
\begin{aligned}
& \mathcal{H}_{j}(W)=\left\{\gamma H_{j} \mid \gamma \in \Gamma\right\}, \quad \mathcal{H}(W)=\bigcup_{j=1}^{p} \mathcal{H}_{j}(W), \\
& \mathcal{V}_{j}(W)=v\left(\mathcal{H}_{j}(W)\right), \quad \mathcal{V}(W)=v(\mathcal{H}(W)) .
\end{aligned}
$$

Since $W$ is not equal to $\mathbf{0}$, it has a non-zero entry; thus $\mathcal{V}(W) \neq \emptyset$. Let $\mathcal{C}(W)$ be the closed convex hull of $\mathcal{V}(W)$ in $\mathbb{M}^{n+1}$, that is, $\mathcal{C}(W)$ is the intersection of closed half spaces of $\mathbb{M}^{n+1}$ which contain $\mathcal{V}(W)$. Then, by Lemma 3.4 of [1], each ray in $T_{+}$from the origin intersects $\partial \mathcal{C}(W)$ at precisely one point. Thus the faces of $\mathcal{C}(W)$ determine a decomposition $\widetilde{\mathcal{D}}(W)$ of $\mathbb{H}^{n}$ by the radial projection from the origin. 
Since $\mathcal{V}(W)$ is invariant under the action of $\Gamma$, so is $\widetilde{\mathcal{D}}(W)$. Thus we can define the decomposition $\mathcal{D}(W)$ by $\widetilde{\mathcal{D}}(W) / \Gamma$. One of the results in [1] states:

Theorem 2.1 ([1]). If $W$ has no zero entry, that is, if $W \in\left(\mathbb{R}_{+}\right)^{p}$, then $\mathcal{D}(W)$ is an ideal polyhedral decomposition with the property $\mathcal{D}(t W)=\mathcal{D}(W)(t>0)$.

We prove the following theorem in the last section.

Theorem 2.2. For $W \in\left(\mathbb{R}_{\geq 0}\right)^{p}-\{\mathbf{0}\}$ which may have zero entries, there exists

$$
\phi: \mathcal{P}_{1} \amalg \cdots \amalg \mathcal{P}_{n_{P}} \amalg \mathcal{Q}_{1} \amalg \cdots \amalg \mathcal{Q}_{n_{Q}} \rightarrow M
$$

with the following properties:

(1) $\phi: \mathcal{P}_{1} \amalg \cdots \amalg \mathcal{P}_{n_{P}} \amalg \mathcal{Q}_{1} \amalg \cdots \amalg \mathcal{Q}_{n_{Q}} \rightarrow M$ is unchanged even if the weight is multiplied by a positive number.

(2) Each $\mathcal{P}_{j}\left(j=1, \ldots, n_{P}\right)$ is a finite sided convex ideal polyhedron of dimension $n$.

(3) Each $\mathcal{Q}_{j}\left(j=1, \ldots, n_{Q}\right)$ is an $n$-dimensional complete hyperbolic manifold with boundary consisting of a finite number of finite sided ideal polyhedra.

(4) The map $\phi$ is an onto continuous map.

(5) The restriction of $\phi$ to each $\mathcal{P}_{j}$ or $\mathcal{Q}_{j}$ is a local isometry and maps the interior injectively.

(6) The images of $\mathcal{P}_{j}$ 's and $\mathcal{Q}_{j}$ 's do not intersect in the interior.

(7) The image of the interior of each $\mathcal{Q}_{j}$ contains a cusp with weight 0 and is homotopy equivalent to the cusp.

In the rest of this section, we characterize the polyhedra in $\widetilde{\mathcal{D}}(W)$ (see Proposition 2.7). At first, notice that the following lemma can be proved by the argument in the proof of Proposition 3.5 of $[1]$. For a convex set $C \subset \mathbb{M}^{n+1}$ and a half space $A \subset \mathbb{M}^{n+1}$, if $C \subset A$ and $\partial C \cap \partial A \neq \emptyset$, we say that $A$ is a supporting half space of $C$.

Lemma 2.3. Let $a$ be a point in $\mathbb{M}^{n+1}$ and $A=\left\{x \in \mathbb{M}^{n+1} \mid\langle x, a\rangle \leq-1\right\}$. If $A$ is a supporting half space of $\mathcal{C}(W)$, then a is contained in $T_{+} \cup L_{+}$. Moreover, a is contained in $L_{+}$if and only if it is a parabolic fixed point of $\Gamma$ corresponding to a cusp with weight 0 .

Note that, since $\mathcal{C}(W)$ is closed, $\partial \mathcal{C}(W)$ is the union of faces of $\mathcal{C}(W)$. However, in contrast with Proposition 3.5 of [1, some faces of $\mathcal{C}(W)$ may be infinite sided polyhedra. To characterize such polyhedra, we need more observation.

The following lemma is a generalization of a well-known proposition in the theory of polytopes. Since $\mathcal{C}(W)$ is the closed convex hull of an infinite point set, it is not a trivial consequence of the general theory.

Lemma 2.4. Let $a$ be a point in $\mathbb{M}^{n+1}$ and $A=\left\{x \in \mathbb{M}^{n+1} \mid\langle x, a\rangle \leq-1\right\}$. If $A$ is a supporting half space of $\mathcal{C}(W)$, then $\partial A \cap \mathcal{C}(W)$ is the closed convex hull of $\partial A \cap \mathcal{V}(W)$. Moreover, $\partial A \cap \mathcal{C}(W)$ is a finite sided polyhedron if and only if $a \in T_{+}$.

Proof. By Lemma 2.3, $a$ is contained in $T_{+} \cup L_{+}$. First, assume that $a \in T_{+}$. Then the intersection of $\partial A$ and $L_{+}$is a compact set. Thus $\partial A \cap L_{+}$has a compact neighborhood in $\mathbb{M}^{n+1}$. Since $\mathcal{V}(W)$ is discrete in $\mathbb{M}^{n+1}$ by Theorem 2.4 of [1], the compact neighborhood of $\partial A \cap L_{+}$contains only a finite number of elements of $\mathcal{V}(W)$. Thus the intersection of $\mathcal{V}(W)$ and a sufficiently small deformation of $\partial A$ 
is contained in this finite set of points. Hence we can easily see that $\partial A \cap \mathcal{C}(W)$ is the closed convex hull of $\partial A \cap \mathcal{V}(W)$ and is a finite sided polyhedron.

Next, assume that $a \in L_{+}$. Then, by Lemma 2.3, $a$ is stabilized by a parabolic subgroup of $\Gamma$ corresponding to a cusp with weight 0 . Since $\langle\gamma x, a\rangle=\left\langle x, \gamma^{-1} a\right\rangle=$ $\langle x, a\rangle$ for any $\gamma \in \operatorname{Stab}(\Gamma, a)$ and $x \in \mathbb{M}^{n+1}, \partial A$ is stabilized by $\operatorname{Stab}(\Gamma, a)$. Thus $\partial A \cap \mathcal{C}(W)$ is stabilized by the infinite group $\operatorname{Stab}(\Gamma, a)$. The following claim holds.

Claim 2.5. Suppose that a half plane $\alpha$ of $\partial A$ contains $\partial A \cap \mathcal{V}(W)$. Then $\alpha$ is the intersection of $\partial A$ and some half space $B$ of $\mathbb{M}^{n+1}$ containing $\mathcal{V}(W)$.

Before proving Claim 2.5, we see that the proof of Lemma 2.4 is completed by using the claim. From the definition, it is obvious that the closed convex hull of $\partial A \cap \mathcal{V}(W)$ is contained in $\partial A \cap \mathcal{C}(W)$. On the other hand, suppose that a point $x \in \partial A$ is not contained in the closed convex hull of $\partial A \cap \mathcal{V}(W)$. Then there is a half plane $\alpha$ of $\partial A$ such that $\alpha \supset \partial A \cap \mathcal{V}(W)$ and $\alpha \not \supset x$. By Claim 2.5, there is a half space $B$ of $\mathbb{M}^{n+1}$ such that $B \supset \mathcal{V}(W)$ and $B \cap \partial A=\alpha$. Since $x$ is not contained in $B$, it is not contained in $\mathcal{C}(W)$. Thus $\partial A \cap \mathcal{C}(W)$ is contained in the closed convex hull of $\partial A \cap \mathcal{V}(W)$. Hence $\partial A \cap \mathcal{C}(W)$ is equal to the closed convex hull of $\partial A \cap \mathcal{V}(W)$.

Let $v$ be a vertex of $\partial A \cap \mathcal{C}(W)$. Since $\partial A \cap \mathcal{C}(W)$ is stabilized by $\operatorname{Stab}(\Gamma, a)$, the point $\gamma v(\gamma \in \operatorname{Stab}(\Gamma, a))$ is also a vertex of $\partial A \cap \mathcal{C}(W)$. Since $\operatorname{Stab}(\Gamma, a)$ is an infinite group, the hull is an infinite sided polyhedron. This completes the proof.

Proof of Claim 2.5. Let $S$ be the $n$-dimensional vector subspace of $\mathbb{M}^{n+1}$ which contains $\partial \alpha$, and $s_{1}, \ldots, s_{n}$ be points in $\partial \alpha$ such that $\left\{s_{1}, \ldots, s_{n}\right\}$ is a basis of $S$. Now, assume that $a \in S$. Then we can see that the normal vector $u$ of $S$ satisfies $\langle u, u\rangle \geq 0$. If $\langle u, u\rangle=0$, then $a$ is also a normal vector of $S$, and hence $\partial A \cap S=\emptyset$. This contradicts the definition of $S$. If $\langle u, u\rangle>0$, then the intersection of $S$ and $\mathbb{H}^{n}$ is an $(n-1)$-dimensional totally geodesic hyperplane which contains $[a]$ in its boundary, where [.] denotes the projection from $L_{+}$onto $\partial \mathbb{H}^{n}$. On the other hand, since $\partial A \cap \mathcal{V}(W)$ is stabilized by $\operatorname{Stab}(\Gamma, a)$, it is impossible that the subset $[\partial A \cap \mathcal{V}(W)]$ of $\partial \mathbb{H}^{n}$ is contained in one side of such a plane, a contradiction. Thus $a$ is not contained in $S$, and hence the set $\left\{s_{1}, \ldots, s_{n}, a\right\}$ is a basis of $\mathbb{M}^{n+1}$.

Choose $a^{\prime}$ so that the plane $\left\{x \in \mathbb{M}^{n+1} \mid\left\langle x, a+a^{\prime}\right\rangle=-1\right\}$ contains $\partial \alpha$. Then $\left\langle s_{1}, a+a^{\prime}\right\rangle=\cdots=\left\langle s_{n}, a+a^{\prime}\right\rangle=-1$. Since $\partial A$ also contains $\partial \alpha,\left\langle s_{1}, a\right\rangle=\cdots=$ $\left\langle s_{n}, a\right\rangle=-1$. Thus we have $\left\langle s_{1}, a^{\prime}\right\rangle=\cdots=\left\langle s_{n}, a^{\prime}\right\rangle=0$. Therefore the set of such $a^{\prime}$ forms a one-dimensional vector subspace of $\mathbb{M}^{n+1}$. Since $\left\{s_{1}, \ldots, s_{n}, a\right\}$ is a basis of $\mathbb{M}^{n+1},\left\langle a, a^{\prime}\right\rangle \neq 0$ for any such $a^{\prime} \neq 0$. Since

$$
\left\langle a+a^{\prime}, a+a^{\prime}\right\rangle=\langle a, a\rangle+2\left\langle a, a^{\prime}\right\rangle+\left\langle a^{\prime}, a^{\prime}\right\rangle=2\left\langle a, a^{\prime}\right\rangle+\left\langle a^{\prime}, a^{\prime}\right\rangle,
$$

there exists $a_{0}^{\prime}$ in the one-dimensional subspace formed by $a^{\prime}$ such that $\left\langle a+\epsilon a_{0}^{\prime}\right.$, $\left.a+\epsilon a_{0}^{\prime}\right\rangle<0$ for any $0<\epsilon<1$.

Let $B$ be the half space of $\mathbb{M}^{n+1}$ which is bounded by a plane considered in the preceding paragraph and does not contain the origin. To be more precise, put $B=\left\{x \in \mathbb{M}^{n+1} \mid\left\langle x, a+\epsilon a_{0}^{\prime}\right\rangle \leq-1\right\}$. In the following, we observe that for a sufficiently small $\epsilon>0, B$ has the desired properties, that is, $\partial A \cap B=\alpha$ and $B \supset \mathcal{V}(W)$. By the definition of $B$, we can see that $\partial B \cap \partial A=\partial \alpha$. Since $a+\epsilon a_{0}^{\prime}$ is contained in $T_{+}$, the intersection of $L_{+}$and the complement of $B$ is contained in a compact subset of $\mathbb{M}^{n+1}$. On the other hand, since $\partial A \cap \mathcal{V}(W)$ is stabilized by the infinite group $\operatorname{Stab}(\Gamma, a), \partial A \cap \mathcal{V}(W)$ consists of infinite points. Using this 
fact and the fact that $\mathcal{V}(W)$ is discrete in $\mathbb{M}^{n+1}$, we can see that $\partial A \cap \mathcal{V}(W)$ is not contained in a compact set. Thus $B \cap \partial A$ is equal to $\alpha$.

Since $\mathcal{V}(W)$ is discrete in $\mathbb{M}^{n+1}$, and since the intersection of $L_{+}$and the complement of $B$ is contained in a compact subset of $\mathbb{M}^{n+1}$, there are at most a finite number of points of $\mathcal{V}(W)$ which are not contained in $B$. Since $B \supset \alpha \supset \partial A \cap \mathcal{V}(W)$, those points are contained in the interior of $A$. Since $B$ is a small deformation of $A$ depending only on $\epsilon$, we can find a sufficiently small $\epsilon>0$ such that there are no such points, that is, $B \supset \mathcal{V}(W)$. This completes the proof.

To make a detailed observation, we need additional definitions. Let $x$ be a point in $\mathbb{H}^{n}$ and $H$ a horoball in $\mathbb{H}^{n}$. The distance $d(x, H)$ between $x$ and $H$ is defined by

$$
d(x, H)=\left\{\begin{array}{l}
d(x, \partial H), \quad \text { if } x \notin H, \text { and } \\
-d(x, \partial H), \quad \text { if } x \in H .
\end{array}\right.
$$

Then the following lemma holds.

Lemma 2.6 ([2, Lemma 3.2]). For a point $x \in \mathbb{H}^{n}$ and a horoball $H \subset \mathbb{H}^{n}$,

$$
d(x, H)=\log (-\langle x, v(H)\rangle) .
$$

Let $\mathcal{K}$ be a set of horoballs. For $x \in \mathbb{H}^{n}$ and a horoball $H \in \mathcal{K}$, we say that $H$ is a nearest horoball to $x$ in $\mathcal{K}$ if

$$
d(x, H)=\min \{d(x, K) \mid K \in \mathcal{K}\} .
$$

We denote by $\mathcal{N}(x, \mathcal{K})$ the set of nearest horoballs to $x$ in $\mathcal{K}$.

For $u \in \partial \mathbb{H}^{n}$ and a set of horoballs $\mathcal{K}$, the highest horoballs in $\mathcal{K}$ with respect to $u$ are defined as follows: First, identify $\mathbb{H}^{n}$ with the upper half space model by a map which sends $u$ to $\infty$. The highest horoballs in $\mathcal{K}$ with respect to $u$ are the horoballs in $\mathcal{K}$ with the largest Euclidean height except for the horoball centered at $u$.

We can characterize the polyhedra in $\widetilde{\mathcal{D}}(W)$ as follows.

Proposition 2.7. Let $U$ be a set of points in $\partial \mathbb{H}^{n}$. The ideal polyhedron $\mathcal{P}$ spanned by $U$ is a polyhedron in $\widetilde{\mathcal{D}}(W)$ if and only if one of the following is satisfied:

(i) There is $x_{0} \in \mathbb{H}^{n}$ such that the set of centers of the nearest horoballs to $x_{0}$ in $\mathcal{H}(W)$ is equal to $U$, and $U$ is a finite set.

(ii) There is a parabolic fixed point $u_{\infty} \in \partial \mathbb{H}^{n}$ of $\Gamma$ corresponding to a cusp with weight 0 such that the set of centers of the highest horoballs in $\mathcal{H}(W)$ with respect to $u_{\infty}$ is equal to $U$.

Proof. First, suppose that $\mathcal{P}$ is a polyhedron in $\widetilde{\mathcal{D}}(W)$ spanned by $U$. Let $A=$ $\left\{x \in \mathbb{M}^{n+1} \mid\langle x, a\rangle \leq-1\right\}$ be a supporting half space of $\mathcal{C}(W)$ such that $\partial A \cap \mathcal{C}(W)$ projects onto $\mathcal{P}$ by the radial projection from the origin. Then, by Lemma 2.3 $a \in T_{+} \cup L_{+}$.

Assume that $a \in T_{+}$. Then, by Lemma 2.4] $\partial A \cap \mathcal{C}(W)$ is a finite sided polyhedron, and hence $U$ is a finite set. Put $x_{0}=a / \sqrt{-\langle a, a\rangle}$. Then $x_{0} \in \mathbb{H}^{n}$ and $A=\left\{x \in \mathbb{M}^{n+1} \mid\left\langle x_{0}, x\right\rangle \leq-1 / \sqrt{-\langle a, a\rangle}\right\}$. By Lemma 2.6 and the definition of $A$, we can see that $U$ is the set of centers of the nearest horoballs to $x_{0}$ in $\mathcal{H}(W)$.

Assume that $a \in L_{+}$. Put $H=v^{-1}(2 a)$ and $u_{\infty}=[a] \in \partial \mathbb{H}^{n}$. Then $H$ is a horoball with center $u_{\infty}$. We can see by the definition of $A$ that $\langle v, v(H)\rangle \leq-2$ for any $v \in \mathcal{V}(W)$ and the equality holds if and only if $[v] \in U$. This is equivalent to 
the condition that the horoballs in $\mathcal{H}(W)$ which intersect $H$ are those with centers in $U$ and are tangent to $H$. Hence, the highest horoballs in $\mathcal{H}(W)$ with respect to $u_{\infty}$ are those with centers in $U$.

Next, suppose that a subset $U$ of $\partial \mathbb{H}^{n}$ satisfies condition (i) or (ii) of this proposition. Assume that condition (i) is satisfied. Then put $a=-x_{0} /\left\langle x_{0}, v(H)\right\rangle$ for arbitrary $H \in \mathcal{N}\left(x_{0}, \mathcal{H}(W)\right)$ and $A=\left\{x \in \mathbb{M}^{n+1} \mid\langle x, a\rangle \leq-1\right\}$. Then, by Lemma 2.6, we can see that $\mathcal{V}(W) \subset A$ and $[\mathcal{V}(W) \cap \partial A]=U$. Thus, by Lemma 2.4, the polyhedron spanned by $U$ is contained in $\widetilde{\mathcal{D}}(W)$.

Assume that condition (ii) is satisfied. Let $H$ be a highest horoball in $\mathcal{H}(W)$ with respect to $u_{\infty}$. Let $a$ be a point in $L_{+}$such that $[a]=u_{\infty}$ and that $\langle v(H), a\rangle=-2$. Then each highest horoball in $\mathcal{H}(W)$ with respect to $u_{\infty}$ is tangent to the horoball $v^{-1}(a)$. Put $A=\left\{x \in \mathbb{M}^{n+1} \mid\langle x, a\rangle \leq-2\right\}$. Then we can see that $\mathcal{V}(W) \subset A$ and $[\mathcal{V}(W) \cap \partial A]=U$. Thus, by Lemma 2.4, the polyhedron spanned by $U$ is contained in $\widetilde{\mathcal{D}}(W)$.

\section{Finiteness of the horoball pattern}

Propositions 3.2 and 3.3 are the key for the proof of Theorems 1.1 and 2.2 Let $W=\left(w_{1}, \ldots, w_{p}\right)$ be an element of $\left(\mathbb{R}_{\geq 0}\right)^{p}-\{\mathbf{0}\}$. For the rest of this paper, we will observe the following convention:

Convention 3.1. For a set of horoballs $\mathcal{K}(W) \subset \mathcal{H}(W)$ and $W^{\prime} \in\left(\mathbb{R}_{\geq 0}\right)^{p}-\{\mathbf{0}\}$, $\mathcal{K}\left(W^{\prime}\right)$ is the set of horoballs in $\mathcal{H}\left(W^{\prime}\right)$ which share the centers with horoballs in $\mathcal{K}(W)$.

Proposition 3.2. Suppose that the $j$-th entry of $W$ is equal to 0 . Let $u_{\infty} \in \partial \mathbb{H}^{n}$ be a parabolic fixed point of $\Gamma$ corresponding to the $j$-th cusp. For $W^{\prime} \in\left(\mathbb{R}_{\geq 0}\right)^{p}-\{\mathbf{0}\}$, denote by $H_{\infty}\left(W^{\prime}\right)$ the horoball in $\mathcal{H}\left(W^{\prime}\right)$ with center $u_{\infty}$ if the $j$-th entry of $W^{\prime}$ is not equal to 0 . Then there are a horoball $H_{j}$ with center $u_{\infty}$, a neighborhood $N_{j}$ of the weight $W$ and subsets $\mathcal{K}_{1}^{(j)}(W), \ldots, \mathcal{K}_{k_{j}}^{(j)}(W), \mathcal{L}_{1}^{(j)}(W), \ldots, \mathcal{L}_{l_{j}}^{(j)}(W)$ of $\mathcal{H}(W)$ with the following properties:

(1) For any $W^{\prime} \in N_{j}$ and $x \in H_{j}$, there are $\gamma \in \operatorname{Stab}\left(\Gamma, H_{j}\right)$ and $k \in\left\{1, \ldots, k_{j}\right\}$ such that $\mathcal{N}\left(\gamma x, \mathcal{H}\left(W^{\prime}\right)\right)$ is equal to $\mathcal{K}_{k}^{(j)}\left(W^{\prime}\right)$ or $\mathcal{K}_{k}^{(j)}\left(W^{\prime}\right) \cup\left\{H_{\infty}\left(W^{\prime}\right)\right\}$.

(2) For any $W^{\prime} \in N_{j}$, there is $l \in\left\{1, \ldots, l_{j}\right\}$ such that the set of highest horoballs in $\mathcal{H}\left(W^{\prime}\right)$ with respect to $u_{\infty}$ is equal to $\mathcal{L}_{l}^{(j)}\left(W^{\prime}\right)$.

Proposition 3.3. For any compact set $K$ in $\mathbb{H}^{n}$, there are a neighborhood $N$ of $W$ and a finite subset $\mathcal{M}(W)$ of $\mathcal{H}(W)$ such that $\mathcal{N}\left(x, \mathcal{H}\left(W^{\prime}\right)\right) \subset \mathcal{M}\left(W^{\prime}\right)$ for any $x \in K$ and $W^{\prime} \in N$.

Proof of Proposition 3.2. Identify $\mathbb{H}^{n}$ with the upper half space model by an isometry which sends $u_{\infty}$ to $\infty$. Since $M$ is of finite volume, $\operatorname{Stab}(\Gamma, \infty)$ acts on the horizontal $(n-1)$-plane with height 0 by Euclidean isometries with a compact quotient. Since $\mathcal{V}(W)$ is discrete in $\mathbb{M}^{n+1}$, the set of Euclidean heights of horoballs in $\mathcal{H}(W)$ is bounded above and discrete in $\mathbb{R}_{+}$. Let $\mathcal{I}(W)$ be the set of highest horoballs in $\mathcal{H}(W)$.

For a point $x=\left(x^{\prime}, t\right)$ in the upper half space $\mathbb{R}^{n-1} \times \mathbb{R}_{+}$and a positive number $R$, we define the $(n-1)$-dimensional ball $B_{h}(x, R)$ in the horizontal plane with height 0 by $B_{h}(x, R)=\left\{\left(x^{\prime \prime}, 0\right)|| x^{\prime \prime}-x^{\prime} \mid \leq R\right\}$, where $|\cdot|$ is the Euclidean norm. Since $\operatorname{Stab}(\Gamma, \infty)$ acts on the horizontal $(n-1)$-plane with height 0 by Euclidean isometries with a compact quotient, we can find a positive number $R_{0}$ with the 
following property: For any $W^{\prime} \in\left(\mathbb{R}_{\geq 0}\right)^{p}-\{\mathbf{0}\}$ and for a point $x$ in the upper half space, $B_{h}\left(x, R_{0}\right)$ contains the center of some highest horoball in $\mathcal{H}\left(W^{\prime}\right)$.

Let $\delta$ be the difference between the largest and the second largest Euclidean heights of the horoballs in $\mathcal{H}(W)$. Let $h_{1}\left(W^{\prime}\right)$ (resp. $h_{2}\left(W^{\prime}\right)$ ) be the Euclidean height of the highest horoballs in $\mathcal{I}\left(W^{\prime}\right)\left(\right.$ resp. $\left.\mathcal{H}\left(W^{\prime}\right)-\mathcal{I}\left(W^{\prime}\right)\right)$. Then there exists a compact neighborhood $N_{j}$ of $W$ such that $h_{1}\left(W^{\prime}\right)-h_{2}\left(W^{\prime}\right)>\delta / 2$ for any $W^{\prime} \in N_{j}$. In particular, the highest horoballs in $\mathcal{H}\left(W^{\prime}\right)$ with respect to $u_{\infty}$ are contained in $\mathcal{I}\left(W^{\prime}\right)$ for any $W^{\prime} \in N_{j}$. Let $\mathcal{I}_{0}(W)$ be a system of representatives up to the action of $\operatorname{Stab}(\Gamma, \infty)$ on $\mathcal{I}(W)$. Since $\mathcal{I}_{0}(W)$ is a finite set, the number of subsets of $\mathcal{I}_{0}(W)$ is also finite. For each subset, consider the set of horoballs equivalent to the ones in this subset up to the action of $\operatorname{Stab}(\Gamma, \infty)$. Let $\mathcal{L}_{1}^{(j)}(W), \ldots, \mathcal{L}_{l_{j}}^{(j)}(W)$ be such sets. Then the set of highest horoballs in $\mathcal{H}\left(W^{\prime}\right)$ with respect to $u_{\infty}$ is equal to some $\mathcal{L}_{l}^{(j)}\left(W^{\prime}\right)$ for any $W^{\prime} \in N_{j}$. Thus assertion (2) holds.

Put $h_{0}=\max \left\{h_{1}\left(W^{\prime}\right), h_{2}\left(W^{\prime}\right)+\delta / 4+R_{0}^{2} / \delta \mid W^{\prime} \in N_{j}\right\}$. We shall denote by $B(x, R)$ the $n$-dimensional Euclidean ball in the upper half space with center $x$ and radius $R$. Let $x=\left(x^{\prime}, t\right)$ be a point in the upper half space with $t>h_{0}$. For $W^{\prime} \in$ $N_{j}$, assume that $B(x, R)$ intersects a horoball in $\mathcal{H}\left(W^{\prime}\right)-\left(\mathcal{I}\left(W^{\prime}\right) \cup\left\{H_{\infty}\left(W^{\prime}\right)\right\}\right)$. Then $R$ is greater than or equal to $t-h_{2}\left(W^{\prime}\right)$. Thus

$$
\begin{aligned}
R & \geq t-h_{2}\left(W^{\prime}\right)>h_{0}-h_{2}\left(W^{\prime}\right) \\
& \geq h_{2}\left(W^{\prime}\right)+\frac{\delta}{4}+\frac{R_{0}^{2}}{\delta}-h_{2}\left(W^{\prime}\right)=\frac{\delta}{4}+\frac{R_{0}^{2}}{\delta} .
\end{aligned}
$$

On the other hand, we can see that the intersection of $B(x, R)$ and the horizontal $(n-1)$-plane with height $h_{1}\left(W^{\prime}\right)$ is an $(n-1)$-dimensional Euclidean ball with radius $\sqrt{R^{2}-\left(t-h_{1}\left(W^{\prime}\right)\right)^{2}}$. By the inequalities $h_{1}\left(W^{\prime}\right)-h_{2}\left(W^{\prime}\right)>\delta / 2$ and $R \geq t-h_{2}\left(W^{\prime}\right)$, we have $t-h_{1}\left(W^{\prime}\right)<R-\delta / 2$, and hence the radius is greater than $\sqrt{R^{2}-(R-\delta / 2)^{2}}=\sqrt{R \delta-\delta^{2} / 4}>R_{0}$. Thus $B(x, R)$ intersects a horoball in $\mathcal{I}\left(W^{\prime}\right)-\left\{H_{\infty}\left(W^{\prime}\right)\right\}$ in the interior.

Now, we can find the nearest horoballs in $\mathcal{H}\left(W^{\prime}\right)-\left\{H_{\infty}\left(W^{\prime}\right)\right\}$ to a point $x=$ $\left(x^{\prime}, t\right)$ with $t>h_{0}$ as follows: Note that any hyperbolic ball centered at $x$ is a Euclidean ball with center $\left(x^{\prime}, t^{\prime}\right)$ for some $t^{\prime}>t$. Since $t>h_{0} \geq h_{1}\left(W^{\prime}\right)$, there is a sufficiently small positive number $r$ such that the hyperbolic ball with center $x$ and radius $r$ does not intersect any horoball in $\mathcal{H}\left(W^{\prime}\right)-\left\{H_{\infty}\left(W^{\prime}\right)\right\}$. Then increase the radius $r$ gradually until the hyperbolic ball is tangent to some horoball in $\mathcal{H}\left(W^{\prime}\right)-\left\{H_{\infty}\left(W^{\prime}\right)\right\}$. Let $B$ be such a hyperbolic ball. The nearest horoballs to $x$ in $\mathcal{H}\left(W^{\prime}\right)-\left\{H_{\infty}\left(W^{\prime}\right)\right\}$ are those which are tangent to $B$. By the observation in the preceding paragraph and the definition of $R_{0}$, we can see that each horoball which is tangent to $B$ is contained in $\mathcal{I}\left(W^{\prime}\right)$ and that the Euclidean radius of the intersection of $B$ and the horizontal $(n-1)$-plane with height $h_{1}\left(W^{\prime}\right)$ is smaller than $R_{0}$. Since $\operatorname{Stab}(\Gamma, \infty)$ acts on each horizontal $(n-1)$-plane by Euclidean isometries with a compact quotient, it has a compact fundamental domain. Let $\mathcal{I}_{1}(W)$ be the set of horoballs in $\mathcal{I}(W)$ which have centers in the $R_{0}$-neighborhood of the fundamental domain and $\mathcal{K}_{1}^{(j)}(W), \ldots, \mathcal{K}_{k_{j}}^{(j)}(W)$ the subsets of $\mathcal{I}_{1}(W)$. Let $H_{j}$ be the horoball consisting of the points with Euclidean height greater than or equal to $h_{0}$. Then, by the above observation, we can see that for any $W^{\prime} \in N_{j}$ and $x \in H_{j}$, there are $\gamma \in \operatorname{Stab}\left(\Gamma, H_{j}\right)$ and $k \in\left\{1, \ldots, k_{j}\right\}$ such that $\mathcal{N}\left(\gamma x, \mathcal{H}\left(W^{\prime}\right)\right)$ is equal to $\mathcal{K}_{k}^{(j)}\left(W^{\prime}\right)$ or $\mathcal{K}_{k}^{(j)}\left(W^{\prime}\right) \cup\left\{H_{\infty}\left(W^{\prime}\right)\right\}$. Thus assertion (1) holds. 
Proof of Proposition 3.3. By Lemma 2.6, we can see that the centers of horoballs in $\mathcal{N}(x, \mathcal{H}(W))$ and those of $\mathcal{N}(x, \mathcal{H}(t W))$ coincide for any $x \in \mathbb{H}^{n}, W \in\left(\mathbb{R}_{\geq 0}\right)^{p}-\{\mathbf{0}\}$ and $t>0$. Thus we can multiply $W$ by any positive number $t$, without loss of generality. So we assume that $\cup \mathcal{H}(W)$ contains $K$ in its interior. Let $\mathcal{M}(W)$ be the set of horoballs in $\mathcal{H}(W)$ which intersect $K$. Then there is a neighborhood $N$ of $W$ such that $\cup \mathcal{M}\left(W^{\prime}\right)$ contains $K$ in the interior and $\cup \mathcal{M}^{c}\left(W^{\prime}\right)$ does not intersect $X$ for any $W^{\prime} \in N$, where $\mathcal{M}^{c}\left(W^{\prime}\right)$ is the complement of $\mathcal{M}\left(W^{\prime}\right)$ in $\mathcal{H}\left(W^{\prime}\right)$. Thus $\mathcal{N}\left(x, \mathcal{H}\left(W^{\prime}\right)\right) \subset \mathcal{M}\left(W^{\prime}\right)$ for any $x \in K$ and $W^{\prime} \in N$. Since $K$ is compact and $\mathcal{V}(W)$ is discrete in $\mathbb{M}^{n+1}, \mathcal{M}(W)$ is a finite set. This completes the proof.

\section{Proof of Theorems 1.1$]$ and 2.2}

Proof of Theorem 2.2. We define $\mathcal{P}_{1}, \mathcal{P}_{2}, \ldots$ and $\mathcal{Q}_{1}, \ldots, \mathcal{Q}_{n_{Q}}$ as follows:

(i) $\left\{\mathcal{P}_{1}, \mathcal{P}_{2}, \ldots\right\}$ is a system of representatives of the finite sided $n$-dimensional polyhedra in $\widetilde{\mathcal{D}}(W)$ up to the action of $\Gamma$.

(ii) Each $\mathcal{Q}_{j}$ is the quotient of an infinite sided polyhedron in $\widetilde{\mathcal{D}}(W)$ by its stabilizer in $\Gamma$, and $\left\{\mathcal{Q}_{1}, \ldots, \mathcal{Q}_{n_{Q}}\right\}$ is a system of representatives of such manifolds up to the action of $\Gamma$.

By Proposition 2.7 we can define a map

$$
\phi:\left(\mathcal{P}_{1} \amalg \mathcal{P}_{2} \amalg \cdots\right) \amalg\left(\mathcal{Q}_{1} \amalg \cdots \amalg \mathcal{Q}_{n_{Q}}\right) \rightarrow M .
$$

Condition (1) of this theorem is obviously satisfied by the definition.

We prove that the number of ideal polyhedra $\mathcal{P}_{j}$ is finite. Since no ideal polyhedron is contained entirely in a cusp, each $\mathcal{P}_{j}$ intersects any compact core of $M$. Thus it suffices to see that the subcomplex of $\widetilde{\mathcal{D}}(W)$ consisting of the finite sided polyhedra is locally finite in $\mathbb{H}^{n}$. Let $K$ be a compact subset of $\mathbb{H}^{n}$ and $\mathcal{R}_{1}, \mathcal{R}_{2}, \ldots$ the distinct polyhedra in $\widetilde{\mathcal{D}}(W)$ which intersect $K$. Let $A_{j}=\left\{x \in \mathbb{M}^{n+1} \mid\left\langle x, a_{j}\right\rangle \leq-1\right\}$ be a supporting half space of $\mathcal{C}(W)$ such that the face $\partial A_{j} \cap \mathcal{C}(W)$ projects onto $\mathcal{R}_{j}$. Since $K$ is compact, there is a subsequence of $\left\{A_{j}\right\}$ which converges to some half space $A=\left\{x \in \mathbb{M}^{n+1} \mid\langle x, a\rangle \leq-1\right\}$. (We also denote the subsequence by $\left\{A_{j}\right\}$.) To be more precise, the sequence $\left\{a_{j}\right\}$ converges to $a$. By Lemma 2.4 each $a_{j}$ is contained in $T_{+}$. Since $a$ is the limit of $\left\{a_{j}\right\}, a$ is contained in $T_{+} \cup L_{+}$. Assume that $a$ is contained in $T_{+}$. Then the intersection of $\partial A$ and $L_{+}$is a compact set. Thus $\partial A \cap L_{+}$has a compact neighborhood. Note that for any sufficiently large $j, \partial A_{j} \cap L_{+}$is contained in this compact neighborhood. On the other hand, since $\mathcal{V}(W)$ is discrete in $\mathbb{M}^{n+1}$, the intersection of $\mathcal{V}(W)$ and the compact neighborhood consists of finite points. Thus it is impossible that the infinite number of faces $\partial A_{j} \cap \mathcal{C}(W)$ are mutually distinct, a contradiction. Hence, by Lemma 2.3 $a$ is a parabolic fixed point of $\Gamma$ corresponding to a cusp with weight 0 . Since $\left\{a_{j}\right\}$ converges to $a$, by Proposition 2.7(i), there is a sequence $\left\{x_{j}\right\} \subset \mathbb{H}^{n}$ such that the vertices of $\mathcal{R}_{j}$ are the centers of horoballs in $\mathcal{N}\left(x_{j}, \mathcal{H}(W)\right)$, and we can see that $\left\{\pi\left(x_{j}\right)\right\}$ exits the cusp with weight 0 . This is impossible by Proposition 3.2 Thus the number of ideal polyhedra $\mathcal{R}_{1}, \mathcal{R}_{2}, \ldots$ is finite. Hence condition (2) of this theorem is satisfied.

Conditions (3), (4), (5) and (6) are clearly satisfied by the definition.

Finally, we prove that the image by $\phi$ of the interior of each $\mathcal{Q}_{j}$ is homotopy equivalent to the cusp contained in $\phi\left(\mathcal{Q}_{j}\right)$. Let $u_{\infty}$ be a parabolic fixed point of $\Gamma$ such that the convex hull $\widetilde{\mathcal{Q}}_{j}$ of the centers of highest horoballs in $\mathcal{H}(W)$ with respect to $u_{\infty}$ projects to $\mathcal{Q}_{j}$. By the definition of $\widetilde{\mathcal{D}}(W)$ and Proposition [2.7)(ii), 
we can see that $\operatorname{int} \widetilde{\mathcal{Q}}_{j}$ intersects $\gamma\left(\operatorname{int} \widetilde{\mathcal{Q}}_{j}\right)(\gamma \in \Gamma)$ if and only if $\gamma \in \operatorname{Stab}\left(\widetilde{\mathcal{Q}}_{j}, \Gamma\right)$. Since $\operatorname{Stab}\left(\widetilde{\mathcal{Q}}_{j}, \Gamma\right)$ is an image of the fundamental group of the cusp contained in $\phi\left(\mathcal{Q}_{j}\right), \phi\left(\operatorname{int} \mathcal{Q}_{j}\right)=\operatorname{int} \widetilde{\mathcal{Q}}_{j} / \operatorname{Stab}\left(\widetilde{\mathcal{Q}}_{j}, \Gamma\right)$ is homotopy equivalent to the cusp contained in $\phi\left(\mathcal{Q}_{j}\right)$.

Proof of Theorem 1.1. First, we observe that for any $W \in\left(\mathbb{R}_{\geq 0}\right)^{p}-\{\mathbf{0}\}$, there is a neighborhood $N$ of $W$ such that $\left\{\mathcal{D}\left(W^{\prime}\right) \mid W^{\prime} \in N\right\}$ is a finite set. For simplicity, assume that $W=\left(w_{1}, \ldots, w_{q}, \ldots, w_{p}\right)$ satisfies $w_{j} \neq 0$ for $j \in\{1, \ldots, q\}$ and $w_{j}=0$ for $j \in\{q+1, \ldots, p\}$. Let $H_{j}(j=q+1, \ldots, p)$ be the horoball and $N_{j}(j=$ $q+1, \ldots, p)$ the neighborhood of $W$ obtained by Proposition 3.2 Since $w_{j} \neq 0$ for $j \in\{1, \ldots, q\}$, it is easy to see that there are a horoball $H_{j}(j=1, \ldots, q)$ stabilized by a parabolic subgroup of $\Gamma$ corresponding to the $j$-th cusp and a neighborhood $N_{j}(j=1, \ldots, q)$ of $W$ with the following property: For $j \in\{1, \ldots, q\}, x \in H_{j}$ and $W^{\prime} \in N_{j}$, the nearest horoball to $x$ in $\mathcal{H}\left(W^{\prime}\right)$ is the horoball which shares the center with $H_{j}$. Since $M-\bigcup_{j=1}^{p} \pi\left(H_{j}\right)$ is compact, there is a compact set $\widetilde{M}_{0}$ in $\mathbb{H}^{n}$ which projects onto $M-\bigcup_{j=1}^{p} \pi\left(H_{j}\right)$. For this $\widetilde{M}_{0}$, let $N^{\prime}$ be the neighborhood of $W$ obtained by Proposition [3.3. Put $N=\bigcap_{j=1}^{p} N_{j} \cap N^{\prime}$. Then, we can see that $\left\{\mathcal{D}\left(W^{\prime}\right) \mid W^{\prime} \in N\right\}$ is a finite set.

Put $\overline{\mathcal{W}}=\left\{\left(w_{1}, \ldots, w_{p}\right) \mid \sum_{j=1}^{p} w_{j}=1\right\} \subset\left(\mathbb{R}_{\geq 0}\right)^{p}-\{\mathbf{0}\}$. Then, by Theorem 2.2 we have

$$
\left\{\mathcal{D}(W) \mid W \in\left(\mathbb{R}_{\geq 0}\right)^{p}-\{\mathbf{0}\}\right\}=\{\mathcal{D}(W) \mid W \in \overline{\mathcal{W}}\} .
$$

By the above observation, for any $W \in \overline{\mathcal{W}}$, there is a neighborhood $N$ of $W$ such that $\{\mathcal{D}(W) \mid W \in N\}$ is a finite set. Thus we can see that $\{\mathcal{D}(W) \mid W \in \overline{\mathcal{W}}\}$ is a finite set, since $\overline{\mathcal{W}}$ is compact. This completes the proof of Theorem 1.1

\section{REFERENCES}

1. D. B. A. Epstein and R. C. Penner, Euclidean decompositions of noncompact hyperbolic manifolds, J. Diff. Geom. 27 (1988), 67-80. MR 89a:57020

2. M. Sakuma and J. Weeks, The generalized tilt formula, Geom. Dedicata 55 (1995), 115-123. MR 96d:57012

Graduate School of Mathematics, Kyushu University 33, Fukuoka 812-8581, Japan

E-mail address: akiyoshi@math.kyushu-u.ac.jp 